\title{
The Latitude of Type I X-Ray Burst Ignition on Rapidly Rotating Neutron Stars
}

\section{Citation}

Cooper, Randall L., and Ramesh Narayan. 2007. "The Latitude of Type I X-Ray Burst Ignition on Rapidly Rotating Neutron Stars." The Astrophysical Journal 657 (1): L29-32. https:// doi.org/10.1086/513077.

\section{Permanent link}

http://nrs.harvard.edu/urn-3:HUL.InstRepos:41384871

\section{Terms of Use}

This article was downloaded from Harvard University's DASH repository, and is made available under the terms and conditions applicable to Other Posted Material, as set forth at http:// nrs.harvard.edu/urn-3:HUL.InstRepos:dash.current.terms-of-use\#LAA

\section{Share Your Story}

The Harvard community has made this article openly available.

Please share how this access benefits you. Submit a story.

Accessibility 
ACCEPTED B Y THE ASTROPHYSiCAL JOURnAL LETTERS

Preprint typeset using $\mathrm{ET}_{\mathrm{E}} \mathrm{X}$ style emulateapj v. 08/22/09

\title{
THE LATITUDE OF TYPE I X-RAY BURST IGNITION ON RAPIDLY ROTATING NEUTRON STARS
}

\author{
RANDALl L. COOPER AND RAMESH NARAYAN \\ Harvard-Smithsonian Center for Astrophysics, 60 Garden Street, Cambridge, MA 02138 \\ Accepted by THE ASTROPHYSICAL JOURNAL LETTERS
}

\begin{abstract}
We investigate the latitude at which type I X-ray bursts are ignited on rapidly rotating accreting neutron stars. We find that, for a wide range of accretion rates $\dot{M}$, ignition occurs preferentially at the equator, in accord with the work of Spitkovsky et al. However, for a range of $\dot{M}$ below the critical $\dot{M}$ above which bursts cease, ignition occurs preferentially at higher latitudes. The range of $\dot{M}$ over which nonequatorial ignition occurs is an increasing function of the neutron star spin frequency. These findings have significant implications for thermonuclear flame propagation, and they may explain why oscillations during the burst rise are detected predominantly when the accretion rate is high. They also support the suggestion of Bhattacharyya \& Strohmayer that non-photospheric radius expansion double-peaked bursts and the unusual harmonic content of oscillations during the rise of some bursts result from ignition at or near a rotational pole.
\end{abstract}

Subject headings: accretion, accretion disks — stars: neutron — X-rays: binaries — X-rays: bursts

\section{INTRODUCTION}

Type I X-ray bursts are thermonuclear explosions that occur on the surfaces of accreting neutron stars in low-mass X-ray binaries (LMXBs) (Babushkina et al. 1975; Grindlay \& Heise 1975; Grindlay et al. 1976; Belian et al. 1976; Woosley \& Taam 1976; Joss 1977; Maraschi \& Cavaliere 1977; Lamb \& Lamb 1977, 1978), and they are triggered by unstable hydrogen or helium burning (for reviews, see Cumming 2004; Strohmayer \& Bildsten 2006). The physics of type I X-ray bursts is generally well understood, and detailed time-dependent one-dimensional models (e.g., Woosley et al. 2004; Fisker et al. 2006) have been rather successful at reproducing the gross characteristics of bursts, such as their fast rise times of $\sim 1 \mathrm{~s}$, durations of $\sim 10-100 \mathrm{~s}$, and recurrence times of a few hours to days.

Nearly all type I X-ray burst models are one-dimensional and thus implicitly assume that matter accretes spherically onto a nonrotating neutron star. Therefore, ignition occurs simultaneously over the entire stellar surface. However, these assumptions are clearly inapplicable to the vast majority of accreting neutron stars. The Rossi X-Ray Timing Explorer has detected highly sinusoidal oscillations with frequencies of 45-1122 Hz during bursts in 17 LMXBs (Strohmayer \& Bildsten 2006 and references therein; Bhattacharyya et al. 2006; Bhattacharyya 2006; Kaaret et al. 2006). It is thought that the burst oscillation frequency corresponds to the neutron star spin frequency (e.g. Strohmayer \& Markwardt 1999), which implies that many neutron stars that exhibit bursts are in fact rapidly rotating. Indeed, Muno et al. (2001, 2004) have shown that the properties of bursts depend sensitively on the neutron star rotation rate, and so rotation must be considered in theoretical burst models. Furthermore, since the time required to accumulate a sufficient amount of fuel to trigger a burst is much greater than the duration of the resulting burst, it is highly unlikely that all of the accreted fuel over the entire neutron star surface will ignite simultaneously (Joss 1978; Shara 1982). Thus, contrary to the assumptions of most theoretical models, ignition almost surely occurs at a point, and the resulting thermonuclear flame subsequently en-

Electronic address: rcooper@cfa.harvard.edu,rnarayan@cfa.harvard.edu gulfs the whole stellar surface in $\sim 1 \mathrm{~s}$, the burst rise time (Fryxell \& Woosley 1982; Bildsten 1995; Spitkovsky et al. 2002, hereafter SLU02). Time-resolved spectroscopy and observations of large amplitude oscillations during the rise of some type I X-ray bursts from rapidly rotating neutron stars support this conclusion (Strohmayer et al. 1997, 1998).

SLU02 investigated the dynamics of localized ignition on a rapidly rotating neutron star. They found that the latitude at which localized ignition occurs is of great importance in determining the burning front propagation speed. It can also affect the stability and nature of zonal flows during the decay phase of bursts (Cumming 2005), the harmonic content of burst rise oscillations (Bhattacharyya \& Strohmayer 2005), and the light curves of non-photospheric radius expansion (PRE) bursts (Bhattacharyya \& Strohmayer 2006a b). SLU02 assert that, due to the reduction of the effective gravitational acceleration, ignition is likely to occur at the equator of a rapidly rotating neutron star. However, recent observations suggest otherwise. Bhattacharyya \& Strohmayer (2005, $2006 \mathrm{a}$ b) argue that the harmonic content of burst rise oscillations and non-PRE double-peaked bursts from the LMXB 4U 1636-536, which harbors a neutron star with spin frequency $\nu=581 \mathrm{~Hz}$, require ignition to oftentimes occur at or near the rotational pole, at least in this source.

In this Letter, we evaluate theoretically the latitude at which type I X-ray bursts on rapidly rotating neutron stars are most probably ignited. In $\$ 2$ we outline the physics that governs the latitude of type I X-ray burst ignition and determine the ignition latitude as a function of the global accretion rate. We discuss our results and conclude in $\$ 3$.

\section{IGNITION LATITUDE}

In this section, we first outline the basic physics that determines the latitude of type I X-ray burst ignition on a rapidly rotating neutron star using a simple model. We then use the global linear stability analysis of Cooper \& Narayan (2005) to carry out more detailed calculations.

\subsection{Basic Physics}

Using the one-zone burst ignition model of Bildsten (1998), we expand upon the work of SLU02 to determine the most probable latitude of burst ignition on the surface of a rapidly 
rotating neutron star. The reader is encouraged to refer to these works for further details. We neglect general relativistic corrections throughout for clarity. Following SLU02, we assume that at all times prior to ignition the accreted plasma is in hydrostatic equilibrium and is at rest in the corotating frame (e.g. Inogamov \& Sunyaev 1999). We presume that the accreted matter spreads in such a way as to minimize the gravitational potential energy of the accreted layer. The base of the accreted layer is therefore an equipotential surface, and so the pressure at the base of the accreted layer is the same at every latitude (e.g., Clayton 1983). Hydrostatic equilibrium thus implies that $P=\Sigma_{\text {layer }}(\lambda) g_{\text {eff }}(\lambda)$ is independent of latitude, where $P$ is the pressure at the base of the accreted layer, $\Sigma_{\text {layer }}$ is the column depth, $g_{\text {eff }}$ is the effective gravitational acceleration, and $\lambda \equiv \pi / 2-\theta$ is the latitude. Thus, $\Sigma_{\text {layer }} \propto g_{\text {eff }}^{-1}$, and so

$$
\dot{\Sigma} \propto g_{\text {eff }}^{-1}
$$

where $\dot{\Sigma}=\dot{\Sigma}(\lambda)$ is the local mass accretion rate per unit area. According to equation (20) of Bildsten (1998), the column depth at which helium ignites $\Sigma_{\text {ign }}$ varies as

$$
\Sigma_{\text {ign }} \propto \dot{\Sigma}^{-1 / 5} g_{\text {eff }}^{-2 / 5}
$$

Combining equations (1) and (2) gives the ignition time as a function of latitude

$$
t_{\text {ign }}(\lambda) \equiv \Sigma_{\text {ign }} / \dot{\Sigma} \propto g_{\text {eff }}^{4 / 5}
$$

Thus $t_{\text {ign }}$, the time it takes to accrete a critical amount of fuel to trigger a type I X-ray burst, is an increasing function of $g_{\text {eff. }}$ Due to centrifugal acceleration, $g_{\text {eff }}$ is lowest at the equator of a rapidly rotating neutron star, which means that $t_{\text {ign }}(\lambda)$ is a minimum at the equator. Therefore, ignition will occur preferentially at the equator (SLU02).

However, nuclear burning on the surface of an accreting neutron star does not always trigger a burst. Both theoretical models (e.g., Fujimoto et al. 1981; Paczyński 1983; Bildsten 1998) and observations (e.g. Cornelisse et al. 2003; Galloway et al. 2006) suggest that there is a local critical accretion rate $\dot{\Sigma}_{\text {crit }}$ above which nuclear burning is stable, and thus bursts do not occur. By equation (1), $\dot{\Sigma}_{\text {crit }}$ is related to the global critical accretion rate $\dot{M}$ such that

$$
\dot{\Sigma}_{\text {crit }} \propto g_{\text {eff }}^{-1} \dot{M}_{\text {crit }}
$$

where $\dot{M}_{\text {crit }}=\dot{M}_{\text {crit }}(\lambda)$ is defined to be the critical global accretion rate above which nuclear burning is stable at latitude $\lambda$. According to equation (24) of Bildsten (1998),

$$
\dot{\Sigma}_{\text {crit }} \propto g_{\text {eff }}^{1 / 2}
$$

and so

$$
\dot{M}_{\text {crit }} \propto g_{\text {eff }}^{3 / 2}
$$

This implies that, since $g_{\text {eff }}$ is lowest at the equator and increases towards the rotational pole, there exists a range of accretion rates for which nuclear burning is stable near the equator and unstable near the pole. Consequently, for a given $\lambda_{0}$ and corresponding critical accretion rate $\dot{M}_{\text {crit }}\left(\lambda_{0}\right)$, nuclear burning is stable for all $\lambda<\lambda_{0}$ and unstable for all $\lambda>\lambda_{0}$. Clearly, at these accretion rates, bursts will be triggered off the equator. For $\dot{M}>\dot{M}_{\text {crit }}(\pi / 2)$, no bursts are triggered at any latitude. Taking the functional derivative of equation (6)

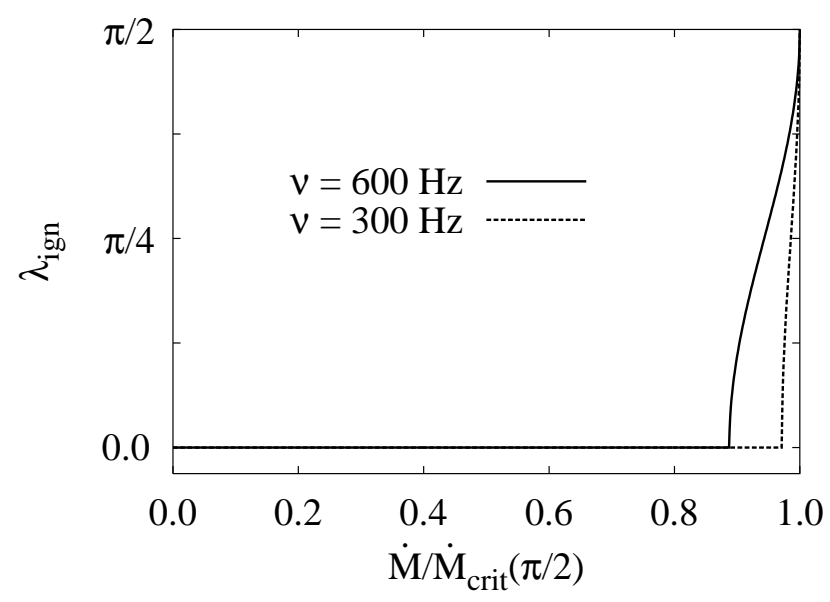

FIG. 1.- Plot shows $\lambda_{\text {ign }}$, the latitude at which type I X-ray bursts ignite on a rapidly rotating neutron star, derived from a one-zone burst model for two stellar rotation frequencies $\nu . M=1.4 M_{\odot}$ and $R=10 \mathrm{~km}$. For a wide range of global accretion rates $\dot{M}$, ignition occurs at the equator $(\lambda=0)$. However, for a range of $\dot{M}$ near the critical rate above which bursts cease, ignition occurs at higher latitudes. The range of $\dot{M}$ over which nonequatorial ignition occurs is $\propto \nu^{2}$.

gives

$$
\frac{\delta \dot{M}_{\text {crit }}}{\dot{M}_{\text {crit }}(\pi / 2)} \approx 0.11\left(\frac{\nu}{600 \mathrm{~Hz}}\right)^{2}\left(\frac{1.4 M_{\odot}}{M}\right)\left(\frac{R}{10 \mathrm{~km}}\right)^{3},
$$

where $\nu, M$, and $R$ are the spin frequency, mass, and radius of the neutron star, respectively. Thus, for a neutron star with spin frequency $\nu=600 \mathrm{~Hz}$, type I X-ray bursts will ignite at the equator for $\dot{M} / \dot{M}_{\text {crit }}(\pi / 2) \lesssim 0.89$ and off of the equator for $0.89 \lesssim \dot{M} / \dot{M}_{\text {crit }}(\pi / 2)<1$. Within the latter range of accretion rates, the latitude at which ignition occurs is given by

$\lambda_{\text {ign }}= \begin{cases}0, & \dot{M}<\dot{M}_{\text {crit }}(0), \\ \arccos \left[\frac{\nu_{\mathrm{K}}}{\nu} \sqrt{1-\left(\frac{\dot{M}_{\text {crit }}(\lambda)}{\dot{M}_{\text {crit }}(\pi / 2)}\right)^{2 / 3}}\right], & \dot{M}_{\text {crit }}(0)<\dot{M}<\dot{M}_{\text {crit }}(\pi / 2),\end{cases}$

where $\nu_{\mathrm{K}}$ is the Keplerian frequency. Figure 1 shows a plot of $\lambda_{\text {ign }}$ as a function of $\dot{M}$. We see that type I X-ray burst ignition on a rapidly rotating neutron star occurs preferentially near the equator for a wide range of $\dot{M}$. However, for a small range of $\dot{M}$ near $\dot{M}_{\text {crit }}$, ignition occurs at higher latitudes because nuclear burning becomes stable near the equator. As equation (7) illustrates, the range of accretion rates over which nonequatorial ignition occurs increases dramatically with $\nu$.

\subsection{Global Linear Stability Analysis}

We now use the global linear stability analysis of Cooper \& Narayan (2005), which is an expanded and improved version of the model of Narayan \& Heyl (2003), to determine the latitude of type I X-ray burst ignition on rapidly rotating neutron stars. We assume that matter accretes at a global rate $\dot{M}$ onto a rapidly rotating neutron star of mass $M=1.4 M_{\odot}$, radius $R=10 \mathrm{~km}$, and spin frequency $\nu=650$ $\mathrm{Hz}$, and it spreads over the stellar surface in the same manner as described in $\$ 2.1$. We set the composition of the accreted matter to be that of the Sun, such that at the neutron star surface the hydrogen mass fraction $X=0.7$, helium mass fraction $Y=0.28$, CNO mass fraction $Z_{\mathrm{CNO}}=0.016$, and heavy 


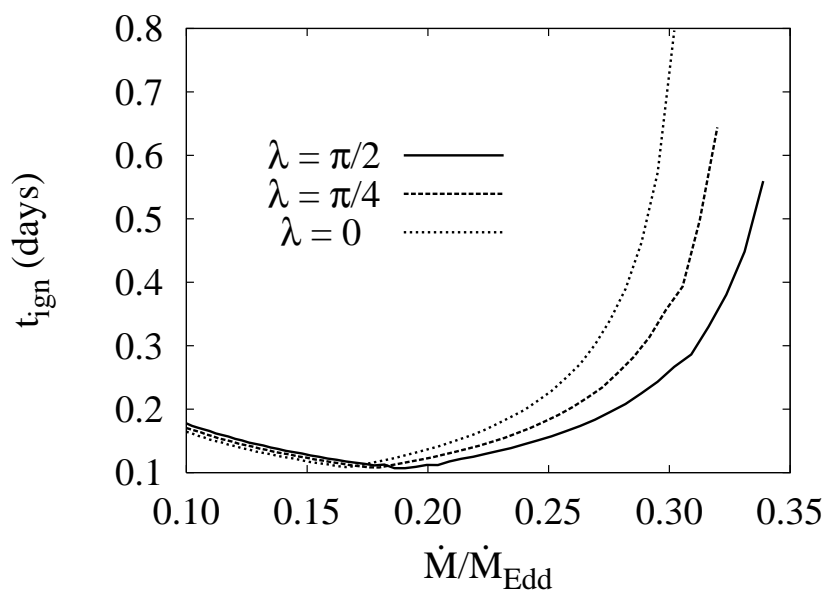

FIG. 2.- Plot shows $t_{\text {ign }}(\lambda)$, the type I X-ray burst ignition timescale, at three different latitudes $\bar{\lambda}$ for a neutron star with spin frequency $\nu=650$ $\mathrm{Hz}, M=1.4 M_{\odot}$, and $R=10 \mathrm{~km}$. For accretion rates $\dot{M} \lesssim 0.16 \dot{M}_{\mathrm{Edd}}, t_{\mathrm{ign}}$ is lowest at the equator, which means that bursts ignite at the equator. For $0.16 \lesssim \dot{M} / \dot{M}_{\text {Edd }} \lesssim 0.19$, the burst ignition latitude increases with $\dot{M}$. For $0.19 \lesssim \dot{M} / \dot{M}_{\text {Edd }} \lesssim 0.3$, although nuclear burning is unstable at all $\lambda$, ignition occurs preferentially near the pole where $t_{\text {ign }}$ is lowest. For $0.3 \lesssim \dot{M} / \dot{M}_{\text {Edd }} \lesssim$ 0.34 , nuclear burning becomes stable at low latitudes, and ignition still occurs preferentially near the pole.

element fraction $Z=0.004$, where $Z$ refers to all metals other than CNO.

We plot the ignition time $t_{\text {ign }}$ as a function of $\dot{M}$ for $\lambda=0$, $\pi / 4$, and $\pi / 2$ in Figure 2 For $\dot{M} \lesssim 0.16 \dot{M}_{\text {Edd }}, t_{\text {ign }}(\lambda)$ is a minimum at the equator, and so bursts ignite preferentially near the equator, in agreement with both SLU02 and equation (3). Here, $\dot{M}_{\text {Edd }}=8.3 \times 10^{17} \mathrm{~g} \mathrm{~s}^{-1}$ denotes the mass accretion rate at which the accretion luminosity is equal to the Eddington limit. For $0.16 \lesssim \dot{M} / \dot{M}_{\text {Edd }} \lesssim 0.19, \lambda_{\text {ign }}$ gradually increases with increasing $\dot{M}$, again in accord with the results of $\$ 2.1$ However, Figure 2 shows a new regime of bursts for $\dot{M} \gtrsim 0.19 \dot{M}_{\mathrm{Edd}}$, the regime of "delayed mixed bursts," which was first identified by Narayan \& Heyl (2003). Delayed mixed bursts are mixed hydrogen and helium bursts triggered by unstable helium burning, and they are preceded by a long period of stable nuclear burning. Using their global linear stability analysis, Narayan \& Heyl found that, within this range of accretion rates, (1) the ignition timescale is an increasing function of $\dot{M}$ (which is consistent with observations, e.g., van Paradijs et al. 1979, 1988; Cornelisse et al. 2003; Remillard et al. 2006; Galloway et al. 2006), and (2) the critical global accretion rate above which delayed mixed bursts occur is an increasing function of the gravitational acceleration $g_{\text {eff. }}$ Thus, for the range $0.19 \lesssim \dot{M} / \dot{M}_{\text {Edd }} \lesssim 0.3$, although nuclear burning is unstable at all latitudes, ignition occurs preferentially near the pole where $t_{\text {ign }}$ is lowest. For $0.3 \lesssim \dot{M} / \dot{M}_{\text {Edd }} \lesssim 0.34$, nuclear burning becomes completely stable at low latitudes, and ignition again occurs preferentially near the pole. For $\dot{M} / \dot{M}_{\text {Edd }} \gtrsim 0.34$, nuclear burning is stable at all latitudes, and no bursts occur.

\section{DISCUSSION AND CONCLUSIONS}

The results of both the simple one-zone model of Bildsten (1998) and the more detailed global linear stability analysis of Cooper \& Narayan (2005) suggest that bursts ignite on the equator at low accretion rates and off of the equator at higher accretion rates. The global linear stability analysis predicts that nonequatorial ignition occurs over a much larger range of $\dot{M}$ - nearly $50 \%$ for $\nu=650 \mathrm{~Hz}$ versus only about $10 \%$ in the one-zone model - and that ignition should occur near the pole for most of this range. The differences in the results of the two models are due to the delayed mixed burst regime, which is predicted in the global linear stability analysis but not in the one-zone model (for an explanation of delayed mixed bursts, see Cooper \& Narayan 2006). In effect, the delayed mixed burst regime significantly extends the range of $\dot{M}$ over which nonequatorial ignition occurs. The global linear stability analysis and the two-zone model of Cooper \& Narayan (2006) agree better with observations than all other current burst models, but this agreement holds only in a time-averaged sense (e.g. Galloway et al. 2006). By this we mean that, while observations imply that the mean ignition time $\left\langle t_{\text {ign }}\right\rangle$ is an increasing function of $\dot{M}$ for $\dot{M} \gtrsim 0.15 \dot{M}_{\text {Edd }}$, which is in accord with the results shown in Figure 2 the ignition time $t_{\text {ign }}$ measured between pairs of bursts observed in nature exhibits significant deviations relative to the mean. Our models cannot account for this behavior. Since the predicted burst ignition latitude is model-dependent, and no current model can successfully reproduce the chaotic behavior of bursts, we have little confidence in the accuracy of our predicted ignition latitudes $\lambda_{\text {ign }}$. However, all theoretical models would predict that bursts ignite preferentially near the equator at low values of $\dot{M}$ and off of the equator at higher $\dot{M}$. This result is very robust.

Recent observations have generated a renewed interest in the latitude of type I X-ray burst ignition. First, non-PRE double peaked bursts have been observed in a few LMXBs (e.g. Sztaino et al. 1985; Penninx et al. 1989; Kuulkers et al. 2002). Bhattacharyya \& Strohmayer (2006a b) argue that ignition near a rotational pole can explain the non-PRE double peaked bursts observed in 4U 1636-536, which contains a neutron star with $\nu=581 \mathrm{~Hz}$. Their simple model of nonequatorial ignition and subsequent thermonuclear flame propagation and temporary stalling near the equator qualitatively reproduces both the light curves and spectral profiles of such bursts. Second, although burst oscillations detected in both the rise and decay are usually quite sinusoidal, Bhattacharyya \& Strohmayer (2005) report evidence for substantial harmonic content in the oscillations during the rise of a burst from 4U 1636-536. They again suggest that nonequatorial ignition and subsequent flame propagation can explain the observations. These authors acknowledged that nonequatorial ignition is at odds with the work of SLU02, who argued for equatorial ignition at all $\dot{M}$. Our result that nonequatorial ignition is more likely when the accretion rate is high naturally reconciles this discrepancy.

That nonequatorial ignition is preferred at higher $\dot{M}$ may explain why oscillations in the rising phase of some bursts on rapidly rotating neutron stars occur predominantly on the banana branch, i.e. when the inferred accretion rate is high (Strohmayer \& Bildsten 2006). This can be understood as follows. SLU02 find that burning fronts propagate much faster near the equator than near the poles. If $\dot{M}$ is low and ignition occurs near the equator, the flame will spread in longitude on a timescale that is much shorter than the burst rise timescale and quickly produce an axisymmetric belt. An observer would detect no oscillations during the rising phase because there is no longitudinal asymmetry. On the other hand, if $\dot{M}$ is high and ignition occurs close to, but not directly at, a rotational 
pole, the flame will spread in the longitudinal direction on a timescale that is comparable to the burst rise timescale. This slow longitudinal spread would create a non-axisymmetric hot spot and hence oscillations. We note that oscillations in the decay phase of some bursts are again observed only when a system is on the banana branch. However, burst oscillations in the decay phase are not well understood theoretically, and we are hesitant to suggest a connection between these oscillations and nonequatorial ignition.

We stress that our results apply only to rapidly rotating, weakly-magnetic neutron stars. Rapid rotation induces a strong latitudinal dependence on the effective gravitational acceleration which restricts burst ignition to a preferred latitude. For a nonrotating (or slowly rotating) neutron star, all physical quantities are independent of $\lambda$ (or nearly so), and ignition could occur at any latitude. Thus, oscillations during the burst rise could occur at any $\dot{M}$ for slowly rotating neutron stars.

Strong magnetic fields may channel and confine accreted matter to some region of the neutron star surface. In this case, ignition is more likely to occur within this confinement region than at a specific latitude. Although some bursts observed from the accreting millisecond pulsar SAX J1808.4-3658 exhibit timing features that Bhattacharyya \& Strohmayer (2006c) suggest originate from midlatitudinal ignition, we speculate that it is due to magnetic confinement.

We thank Josh Grindlay for discussions that motivated this investigation and the referee for useful comments. This work was supported by NASA grant NNG04GL38G.

\section{REFERENCES}

Babushkina, O. P., Bratolyubova-Tsulukidze, L. S., Kudryavtsev, M. I., Melioranskiy, A. S., Savenko, I. A., \& Yushkov, B. Y. 1975, Soviet Astronomy Letters, 1, 32

Belian, R. D., Conner, J. P., \& Evans, W. D. 1976, ApJ, 206, L135

Bhattacharyya, S. 2006, MNRAS, submitted (astro-ph/0605510)

Bhattacharyya, S. \& Strohmayer, T. E. 2005, ApJ, 634, L157

-. 2006a, ApJ, 636, L121

-. 2006b, ApJ, 641, L53

-. 2006c, ApJ, 642, L161

Bhattacharyya, S., Strohmayer, T. E., Markwardt, C. B., \& Swank, J. H. 2006, ApJ, 639, L31

Bildsten, L. 1995, ApJ, 438, 852

Bildsten, L. 1998, in The Many Faces of Neutron Stars, ed. R. Buccheri, J. van Paradijs, \& M. A. Alpar (NATO ASI Ser. C, 515; Dordrecht: Kluwer), 419

Clayton, D. D. 1983, Principles of stellar evolution and nucleosynthesis (Chicago: University of Chicago Press, 1983)

Cooper, R. L. \& Narayan, R. 2005, ApJ, 629, 422

-. 2006, ApJ, 652, 584

Cornelisse, R., in't Zand, J. J. M., Verbunt, F., Kuulkers, E., Heise, J., den Hartog, P. R., Cocchi, M., Natalucci, L., Bazzano, A., \& Ubertini, P. 2003, A\&A, 405, 1033

Cumming, A. 2004, Nuclear Physics B Proceedings Supplements, 132, 435

-. 2005, ApJ, 630, 441

Fisker, J. L., Görres, J., Wiescher, M., \& Davids, B. 2006, ApJ, 650, 332

Fryxell, B. A. \& Woosley, S. E. 1982, ApJ, 261, 332

Fujimoto, M. Y., Hanawa, T., \& Miyaji, S. 1981, ApJ, 247, 267

Galloway, D. K., Muno, M. P., Hartman, J. M., Savov, P., Psaltis, D., \& Chakrabarty, D. 2006, ApJS, submitted (astro-ph/0608259)

Grindlay, J., Gursky, H., Schnopper, H., Parsignault, D. R., Heise, J., Brinkman, A. C., \& Schrijver, J. 1976, ApJ, 205, L127

Grindlay, J. \& Heise, J. 1975, IAU Circ., 2879

Inogamov, N. A. \& Sunyaev, R. A. 1999, Astronomy Letters, 25, 269

Joss, P. C. 1977, Nature, 270, 310

-. 1978, ApJ, 225, L123

Kaaret, P., Prieskorn, Z., in 't Zand, J. J. M., Brandt, S., Lund, N., Mereghetti, S., Gotz, D., Kuulkers, E., \& Tomsick, J. A. 2006, ApJL, submitted (astro-ph/0611716)
Kuulkers, E., Homan, J., van der Klis, M., Lewin, W. H. G., \& Méndez, M. 2002, A\&A, 382, 947

Lamb, D. Q. \& Lamb, F. K. 1977, in Eight Texas Symposium on Relativistic Astrophysics, ed. M. D. Papagiannis (New York: New York Academy of Sciences), 261

Lamb, D. Q. \& Lamb, F. K. 1978, ApJ, 220, 291

Maraschi, L. \& Cavaliere, A. 1977, Highlights of Astronomy, 4, 127

Muno, M. P., Chakrabarty, D., Galloway, D. K., \& Savov, P. 2001, ApJ, 553, L157

Muno, M. P., Galloway, D. K., \& Chakrabarty, D. 2004, ApJ, 608, 930

Narayan, R. \& Heyl, J. S. 2003, ApJ, 599, 419

Paczyński, B. 1983, ApJ, 264, 282

Penninx, W., Damen, E., van Paradijs, J., Tan, J., \& Lewin, W. H. G. 1989, A\&A, 208, 146

Remillard, R. A., Lin, D., Cooper, R. L., \& Narayan, R. 2006, ApJ, 646, 407 Shara, M. M. 1982, ApJ, 261, 649

Spitkovsky, A., Levin, Y., \& Ushomirsky, G. 2002, ApJ, 566, 1018 (SLU02)

Strohmayer, T. \& Bildsten, L. 2006, in Compact Stellar X-Ray Sources, ed.

W. H. G. Lewin and M. van der Klis (Cambridge: Cambridge Univ.

Press), 113

Strohmayer, T. E. \& Markwardt, C. B. 1999, ApJ, 516, L81

Strohmayer, T. E., Zhang, W., \& Swank, J. H. 1997, ApJ, 487, L77

Strohmayer, T. E., Zhang, W., Swank, J. H., White, N. E., \& Lapidus, I. 1998, ApJ, 498, L135

Sztajno, M., van Paradijs, J., Lewin, W. H. G., Trumper, J., Stollman, G., Pietsch, W., \& van der Klis, M. 1985, ApJ, 299, 487

van Paradijs, J., Cominsky, L., Lewin, W. H. G., \& Joss, P. C. 1979, Nature, 280,375

van Paradijs, J., Penninx, W., \& Lewin, W. H. G. 1988, MNRAS, 233, 437

Woosley, S. E., Heger, A., Cumming, A., Hoffman, R. D., Pruet, J., Rauscher, T., Fisker, J. L., Schatz, H., Brown, B. A., \& Wiescher, M. 2004, ApJS, 151, 75

Woosley, S. E. \& Taam, R. E. 1976, Nature, 263, 101 